PAPER

\title{
Clinical characteristics of multiple sclerosis in Västerbotten County in northern Sweden
}

\author{
P Sundström, A Svenningsson, L Nyström, L Forsgren
}

See end of article for

authors' affiliations

........................

Correspondence to: Dr Peter Sundström Department of Neurology, Umeå University Hospital, S-901 85 Umeå, Sweden; peter.sundstrom@ neuro.umu.se

Received 18February 2003 In revised form

24 September 2003

Accepted

25 September 2003
J Neurol Neurosurg Psychiatry 2004;75:711-716. doi: 10.1136/jnnp.2003.012856

Background: Most clinical data for multiple sclerosis are hospital based-that is, derived from patients referred to clinics specialising in the disease.

Objectives: To present data derived from two population based multiple sclerosis populations, an incidence cohort and a prevalence population, from Västerbotten County, northern Sweden.

Methods: The two populations were identified from multiple sources, and case ascertainment was assured through a personal clinical review, including interviews and examination of the patients.

Results: Characteristics at onset for the different clinical subtypes of multiple sclerosis are presented, including the clinical spectrum of the first attack, the anatomical correlation between the first and second attacks, sex distribution, and disability distribution.

Conclusions: Based on the comparison of present and earlier natural history data, multiple sclerosis appears to be a slightly more benign disease than previously recognised.
$\mathrm{P}$ opulation based clinical data on multiple sclerosis are rare. When presented, such data are often derived from available medical records. The reports are usually based on hospital derived case series and may therefore be biased towards the more severe forms of the disease. It is important to obtain clinical information about multiple sclerosis from population based studies as these can provide unbiased natural history data and data on the disease spectrum. Information from incidence cohorts complements cross sectional clinical data from prevalence populations. The incident cohort represents the inherent natural history of the disease, providing input for the prevalence population; the prevalence population pictures the disease and its impact at a given point in time.

In this study we present population based clinical data on multiple sclerosis from a 10 year incidence cohort as well as from a prevalence population from Västerbotten County, northern Sweden.

\section{METHODS}

\section{Material}

Västerbotten County, northern Sweden, is sparsely populated with 259163 inhabitants $\left(4.7 / \mathrm{km}^{2}\right)$ on the prevalence day, 31 December 1997. All the clinical data came from two studies on multiple sclerosis, one on the incidence and one on the prevalence. $^{1}$

Incident cases $(n=133)$ had experienced an onset symptom in agreement with the definition, "defined onset", while residing in the study area any time from 1 January 1988 to 31 December 1997, and were later judged to fulfil the Poser diagnostic criteria for definite or probable multiple sclerosis. ${ }^{2}$

Prevalent cases $(n=399)$ had at least one defined onset symptom before the prevalence day, were residents in the study area on the prevalence day, 31 December 1997, and fulfilled the same Poser criteria at the time of data collection. Seventy seven per cent of the prevalence population were born in the study area and $82 \%$ were resident in the study area at the age of 15. Four prevalent cases were nonEuropean immigrants. Ninety five per cent $(n=127)$ of the incidence population formed part of the prevalence population.
Up to year 2001, data collected from multiple sources were used to make case identification as complete as possible. Case ascertainment and reliability of clinical data were assured from medical records, the results of magnetic resonance imaging (MRI), cerebrospinal fluid (CSF) electrophoresis analysis, and other laboratory and paraclinical tests, together with follow up interviews and neurological examinations including expanded disability status scale (EDSS) assessment. ${ }^{3}$ Further details on methodology, basal epidemiological data, and case ascertainment have been presented previously.

Clinical judgement determined the use of data received from either interviews or medical records when these data were in conflict. If new information emerged from interviews, such as an earlier first attack than was previously recorded, we retrieved the corresponding medical records, if available. Medical records written less than five years after disease onset were available in $92 \%$ of incident cases and $82 \%$ of prevalent cases. Interferon $\beta$ treatment was approved in 1995 in Sweden, but only five cases were treated before the end of the incidence period coinciding with the prevalence day. The use of immunosuppressive treatment other than corticosteroids at relapses was likewise limited to a handful of patients. Clinical outcome was not affected by participation in clinical trials.

\section{Definitions}

A specification of symptoms (table 3 ) to be considered as the first manifestation of relapsing-remitting multiple sclerosis (RRMS) was used to define disease onset, "defined onset", in RRMS cases. ${ }^{4}$ Any preceding symptoms possibly constituting the onset, "possible onset", of RRMS were also recorded. When not specified otherwise, "defined onset" was used for calculations on age at onset and disease duration. The duration, sensory/motor distribution when applicable, and

Abbreviations: DSS, disability status scale; EDSS, expanded disability status scale; PPMS, primary progressive multiple sclerosis; PRMS, progressive relapsing multiple sclerosis; RRMS, relapsing-remitting multiple sclerosis; SPMS, secondary progressive multiple sclerosis 
grade of remission of the "defined onset" attack were recorded. Using the same specification as for "defined onset" symptoms, the second attack, if present, indicating dissemination in time and space was also recorded in RRMS cases. The year of onset of progressive neurological symptoms was recorded for primary progressive (PPMS), secondary progressive (SPMS), and progressive-relapsing multiple sclerosis (PRMS). ${ }^{5}$ If a progressive disease course was preceded by only a "possible onset" attack believed to represent the true onset of multiple sclerosis, the disease course was classified as SPMS, and the year for SPMS onset was regarded as the year of "defined onset". The year of the prevalence day was used for calculation of disease duration. The exception was when clinical data were also analysed, when the year for data collection was used.

\section{Follow up}

As part of the clinical review of cases, a follow up interview was done between August 1997 and December 2001, median year 1998, for 123 of 133 incident cases (93\%) and 364 of 399 prevalent cases $(91 \%)$. In 57 prevalent cases, the interview information was at least partially retrieved from participating relatives or caregivers. Incident cases not participating in the follow up interview had shorter disease duration; however, this was not statistically significant compared with interviewees. The opposite non-significant difference was true for prevalent cases. Follow up neurological examinations including EDSS assessments were undertaken by PS in 95 incident and 324 prevalent cases.

\section{Statistical methods}

SPSS was used for computerisation and statistical analysis. The variables age and duration are skewed; thus only the median has been presented. The Kruskal-Wallis test was used to test the difference between total and partial remission of the first attack. For all other tests of the difference between median values, the median test was applied. The association between duration of first attack and age at onset was analysed using Pearson's product moment correlation coefficient. The association between first and second attacks was analysed by the $\chi^{2}$ test.

\section{RESULTS}

\section{Disease duration}

Year of onset in the prevalence population ranged from 1935 to 1997, and the median (mean) disease duration was 15 (18) years. Median (mean) disease duration was 15 (18) years in RR/SPMS and 12 (15) years in PPMS prevalent cases $(\mathrm{p}=0.008)$.

\section{Age at onset and clinical course}

The clinical course distribution was similar in the incidence and prevalence group (table 1). Ages at onset by sex and clinical course are presented in table 2. Median age at onset was higher for incident cases than for prevalent cases ( $34 v 30$ years (range 14 to $63 \vee 7$ to 68 ); $\mathrm{p}=0.024$ ). There was no significant difference between female and male subjects. Median age at onset for prevalent PPMS cases was 10 years higher than for prevalent RR/SPMS cases (39 $v 29$ years (range 15 to $68 v 7$ to 58 ); $\mathrm{p}<0.001$ ). Median age at "possible onset" was lower than "defined onset" for prevalent RR/ SPMS cases ( $27 v 29$ years). This difference was as great as 29 years in individual cases in the incidence population and 30 years in the prevalence population. Median age at onset for prevalent PRMS cases was 32 years $(n=13$, not shown in the table).

Table 3 describes the distribution of onset attack symptoms among RR/SPMS incident and prevalent cases. In incident $(\mathrm{n}=3)$ and prevalent $(\mathrm{n}=20)$ RR/SPMS cases with multifocal onset, the predominating onset symptom was chosen.

Prevalent cases with total remission had a lower median age at onset ( 29 years, $\mathrm{n}=118$ ) than patients with a partial remission of their first attack ( 32 years, $n=83)(p=0.035)$ (cases with multifocal onset were excluded, and only information in original medical records was used for both). The maximum duration of the onset attack in cases with total remission was 12 months. There was a weak association between duration of first attack and age at onset $(r=0.28$; $\mathrm{p}=0.05$ ), found only in incident cases.

In $39 \%$ of incident and $32 \%$ of prevalent RR/SPMS cases, a "possible onset" symptom preceded the "defined onset". A majority of these symptoms were sensory or motor symptoms not included in table 3-that is, symptoms not clearly involving an entire limb or sensory symptoms with facial or truncal localisation. Visual symptoms (perhaps from an undiagnosed optic neuritis), possible brain stem symptoms, neuropathic pain, dysuria, psychiatric symptoms, and epileptic seizures formed the remainder of symptoms judged to constitute the onset of RRMS.

\section{Second manifestation in RR/SPMS}

The time to the second manifestation of RR/SPMS (attack or progressive symptoms) was analysed in 90 incident cases after excluding cases with only one attack of the symptoms listed in table 3 at follow up $(n=12)$ and cases where the onset of SPMS constituted "defined onset" $(\mathrm{n}=4)$. The median duration (and range) between the first and second definite multiple sclerosis manifestation was 2 ( 0 to 9 ) years. In 15 of the 90 incident cases the second manifestation of multiple sclerosis was the conversion to SPMS.

Prevalent RR/SPMS cases $(n=141)$ having had both their first and second attack in the optic nerve, brain stem/ cerebellum, or spinal cord region (table 3) were analysed separately. Cases with multifocal onset were excluded from this analysis. In all, 40\% more cases than expected were found to have had their first and second attack in the same region (70 actual $v 50.0$ expected, $\mathrm{p}=0.004$ ). This was most pronounced in cases with optic nerve and brain stem/ cerebellar attacks, where there was a $57 \%$ and $74 \%$ increased likelihood of having the second attack in the same respective region. This finding was achieved despite the fact that the second attack had to show dissemination in space. Although non-significant, the same finding was confirmed in incident cases $(n=40)$, for which a $37 \%$ increased likelihood of having the first and second attack in the same anatomical region was found ( 21 actual $v 15.3$ expected, $\mathrm{p}=0.22$ ).

\section{Conversion to SPMS}

Fifty per cent (161 of 325) initially RRMS prevalent cases were known to have entered SPMS at follow up. Median time from the onset attack to SPMS was 10 years (range 0 to 36 , $\mathrm{n}=150$ ) for prevalent cases with known year of SPMS onset. Median age at onset of SPMS for these cases was 42 years (range 14 to 74 ), which did not differ significantly from the age of onset for PPMS prevalent cases.

In 23 cases with a disease duration exceeding 25 years, no conversion to SPMS could be documented. Median EDSS in these patients was 3.5, opposing any significant underestimation of SPMS conversion. The support from CSF and MRI for a diagnosis of multiple sclerosis in these 23 elderly cases was if anything more solid than in the SPMS converted cases with the same disease duration.

The clinical course was or had been RRMS in 106 incident cases (80\%); 27 (25\%) of these had entered SPMS at follow up after a median disease duration of five years. Progression in every incident SPMS case was characterised by sensorymotor symptoms predominating in the lower extremities- 
Table 1 Clinical course in the incidence cohort and prevalence population

\begin{tabular}{|c|c|c|c|c|}
\hline \multirow[b]{2}{*}{ Clinical course } & \multicolumn{2}{|c|}{ Incidence $(n=133)$} & \multicolumn{2}{|c|}{ Prevalence $(n=399)$} \\
\hline & $\%$ & $\mathbf{n}$ & $\%$ & $\mathbf{n}$ \\
\hline Relapsing-remitting/secondary progressive & 80 & 106 & 81 & 325 \\
\hline Primary progressive & 14 & 19 & 12 & 49 \\
\hline Progressive-relapsing & 4.5 & 6 & 3.3 & 13 \\
\hline Uncertain & 1.5 & 2 & 3.0 & 12 \\
\hline
\end{tabular}

that is, a parasyndrome-except for one case with a progressive opticopathy.

\section{PPMS onset symptoms}

Disease onset in PPMS incident cases was compatible with a sensory or motor parasyndrome in all but four patients, three of whom had hemisymptoms initially and one had cerebellar onset symptoms. Except in eight cases, the onset was consistent with a sensory or motor parasyndrome in all PPMS prevalent cases; in six cases hemisymptoms predominated initially, and one each had cerebellar and optic symptoms.

\section{Sex ratio}

The sex ratios (female to male) for all prevalent cases, RR/ SPMS prevalent cases, and PPMS prevalent cases were 1.9,

Table 2 Median age of defined (and possible) onset by sex in clinical course classifiable prevalent $(n=387)$ and incident $(n=131)$ cases

\begin{tabular}{|c|c|c|c|c|c|c|}
\hline & \multicolumn{3}{|c|}{ Incidence database } & \multicolumn{3}{|c|}{ Prevalence database } \\
\hline & Total & Female & Male & Total & Female & Male \\
\hline All cases & $34(32)$ & 34 (32) & 33 (33) & $30(28)$ & 30 (27) & $30(29)$ \\
\hline RRMS & $32(32)$ & 33 (31) & 32 (32) & 29 (27) & $29(26)$ & 29 (27) \\
\hline PPMS & 41 & 44 & 41 & 39 & 39 & 40 \\
\hline
\end{tabular}

PPMS, primary progressive multiple sclerosis; RRMS, relapsing-remitting multiple sclerosis

Table 3 Distribution of defined onset attack symptoms

\begin{tabular}{|c|c|c|c|c|}
\hline \multirow[b]{2}{*}{ Onset symptom } & \multirow{2}{*}{$\begin{array}{l}\text { Incidence (\%) } \\
\text { Total }\end{array}$} & \multicolumn{3}{|c|}{ Prevalence (\%) } \\
\hline & & Total & Female & Male \\
\hline \multicolumn{5}{|l|}{ Optic nerve } \\
\hline Unilateral optic neuritis & 12 & 24 & 26.5 & 20 \\
\hline Acquired monocular colour blindness & 1 & 0.5 & 0.5 & \\
\hline \multicolumn{5}{|l|}{ Brain stem/cerebellar } \\
\hline Oscillopsia & & 0.5 & 0.5 & 1 \\
\hline True binocular diplopia & 12 & 12 & 9 & 17 \\
\hline Unilateral facial palsy* & 1 & 2 & 2 & 2 \\
\hline Acute unilateral diminution of hearing (under age 40 ) & & $\overline{1}$ & $\overline{1}$ & \\
\hline Transient acute non-positional vertigo (under age 40) & 8 & 4 & 5 & 2 \\
\hline \multicolumn{5}{|l|}{ Spinal cord } \\
\hline Myelitis & 32 & 31 & 33 & 28 \\
\hline Lhermittes symptom & 1 & 2 & 2 & 2 \\
\hline Gait ataxia & 4 & 1 & 1 & 1 \\
\hline Unilat dysmetria/intention tremor/incoordination & & 1 & 0.5 & 1 \\
\hline Painless urinary urgency/incontinence in men & & 0.5 & & 1 \\
\hline Painless urinary frequency in men (under age 40$)^{*}$ & & 0.5 & & 1 \\
\hline \multicolumn{5}{|l|}{ Cerebral/unclear } \\
\hline Weakness/paraesthesiae of one entire limb† & 29 & 20 & 19 & 24 \\
\hline Total & 100 & 100 & 100 & 100 \\
\hline Number of cases & 102 & 316 & 218 & 98 \\
\hline \multicolumn{5}{|l|}{ Sensory and/or motor distribution within symptom group } \\
\hline \multicolumn{5}{|l|}{ Myelitis } \\
\hline Pure sensory & 72 & 68 & 68 & 67 \\
\hline Pure motor & 9 & 7 & 7 & 7 \\
\hline Sensory-motor & 19 & 25 & 25 & 26 \\
\hline \multicolumn{5}{|l|}{ Weakness/paraesthesiae of one entire limb $†$} \\
\hline Pure sensory & 56 & 48 & 48 & 48 \\
\hline Pure motor & 17 & 16 & 17 & 13 \\
\hline Sensory-motor & 27 & 36 & 35 & 39 \\
\hline
\end{tabular}

The original list of onset symptoms was slightly modified (Poser, 19954).

*These symptoms had to be followed by one of the other symptoms within two years.

†Hemisymptoms were included in this group. In accordance with original recommendations, transient hemiparesis (under age 40) was regarded as a " $" * \prime \prime$ symptom 
Table 4 The female to male ratio by age at onset and anatomical region of first attack in prevalent cases

\begin{tabular}{|c|c|c|c|c|c|c|c|c|}
\hline \multirow{2}{*}{$\begin{array}{l}\text { Age at } \\
\text { onset } \\
\text { (years) }\end{array}$} & \multicolumn{2}{|c|}{ Optic nerve } & \multicolumn{2}{|c|}{ Brain stem/cerebellar } & \multicolumn{2}{|c|}{ Spinal cord } & \multicolumn{2}{|c|}{ Cerebral/unclear } \\
\hline & Sex ratio & $\mathbf{n}$ & Sex ratio & $\mathbf{N}$ & Sex ratio & $\mathbf{n}$ & Sex ratio & $\mathbf{n}$ \\
\hline 0 to 28 & 2.2 & $28 \mathrm{~F} / 13 \mathrm{M}$ & 1.6 & $21 F / 13 M$ & 3.7 & $44 \mathrm{~F} / 12 \mathrm{M}$ & 1.8 & $18 \mathrm{~F} / 10 \mathrm{M}$ \\
\hline $29+$ & 5.2 & $31 \mathrm{~F} / 6 \mathrm{M}$ & 1.9 & $21 F / 11 M$ & 1.7 & $32 F / 19 M$ & 1.6 & $23 F / 14 M$ \\
\hline
\end{tabular}

F, female; $M$, male.

2.3, and 1.0, respectively. The corresponding figures for incident cases were 1.8, 2.1, and 1.1. The sex ratio in incident and prevalent PPMS cases was the same regardless of age at onset.

We further analysed whether subgrouping cases for age and the anatomical region of the first attack affected the sex ratios. The onset attack symptoms were aggregated into four groups based on anatomical region (table 3): optic nerve, brain stem/cerebellum, spinal cord, and cerebral/unclear. Table 4 shows the female to male ratio by age at onset and anatomical region of first attack for 316 prevalent RR/SPMS cases, for all of which the sex ratio was 2.2.

The two most pronounced deviations in sex ratios were first, that an optic nerve onset was an uncommon presentation in older male subjects, and second, that a spinal cord onset was more frequent in younger female subjects.

\section{Disability}

EDSS was assessed in 389 prevalent and 130 incident cases. We tried not to use assessments done during an exacerbation and, when information in medical records was used, to choose an examination date as close to the prevalence day as possible. Median year for assessment of prevalent cases was $1998,83 \%$ of assessments being performed in 1997 to 1998.

The distribution of EDSS was: 0 to 2.5 in $36 \%(n=139), 3.0$ to 5.5 in $27 \%(n=104), 6.0$ to 7.5 in $21 \%(n=82)$, and 8.0 to 9.5 in $16 \%(n=64)$. EDSS distribution by age group and sex
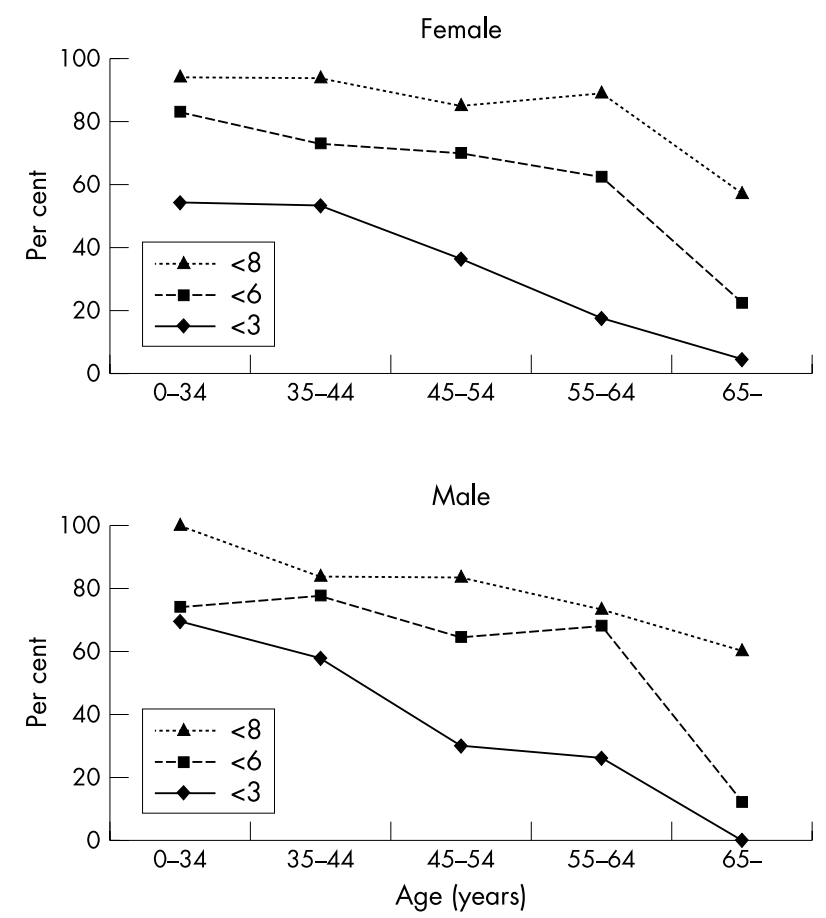

Figure 1 Relative EDSS distribution within age group and sex; data for 390 prevalent cases. is shown in fig 1. Figure 2 shows relative EDSS distribution by disease duration.

Median year for EDSS assessment in incident cases was 1998 (range 1993 to 2001). After a median disease duration of five years (range 0 to 10), the EDSS distribution was: 0 to 2.5 in $62 \%(n=81), 3.0$ to 5.5 in $21 \%(n=27), 6.0$ to 7.5 in $14 \%(n=18)$, and 8.0 to 9.0 in $3 \%(n=4)$.

\section{DISCUSSION}

\section{The onset of multiple sclerosis}

This study provides population based clinical data on onset symptom, second attack, clinical course, and EDSS distribution in an incidence population and a prevalence population. The determination of multiple sclerosis onset is fundamental for this discourse. Onset symptoms in multiple sclerosis have been categorised previously in different ways. To our knowledge the only effort to produce a standard allowing comparisons of epidemiological studies is the list of onset symptoms adopted here. ${ }^{4}$ Some items in the original list were found to be of little or no use. On the other hand, the present "defined onset" criteria did not include all the symptoms judged to represent the true disease onset. Thus nine prevalent and four incident cases were classified as having SPMS in the absence of any "defined onset" attack. In this material, data for both "possible" and "defined" onset are given, the former being more sensitive and the later more specific. The choice of onset definition significantly affected the median age of onset by two years in prevalent RR/SPMS cases. As far as comparison is possible, the present distribution of "defined onset" symptoms appears to be comparable to the findings in other studies. ${ }^{6}$ We believe a standard of onset symptoms is required for a thorough comparison of epidemiological studies of multiple sclerosis.

\section{Age at onset}

Incident cases had a greater age at onset than prevalent cases. Possible explanations are that patients with early onset have a longer interval between the first and second attacks and are
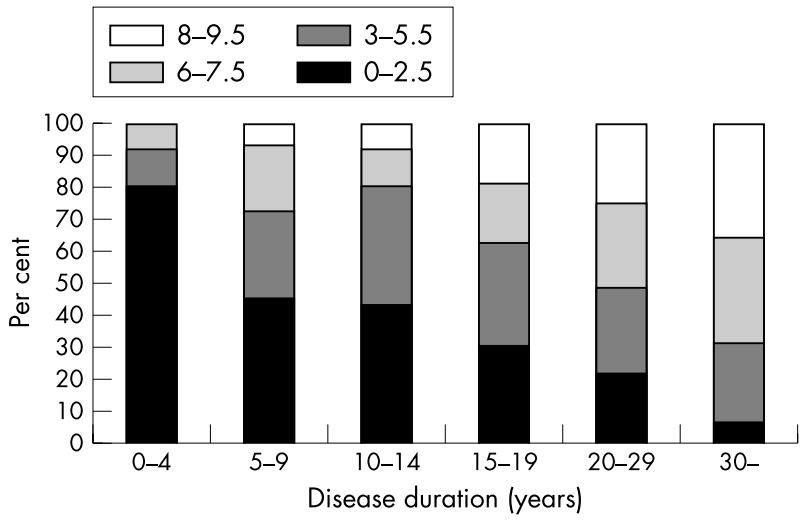

Figure 2 Relative EDSS distribution by disease duration; data for 389 prevalent cases. 
underrecognised in this incidence cohort. Alternatively, cases with early onset, inferring a better prognosis, are overrepresented in the prevalence population because of better survival. The higher prevalence of optic neuritis as an onset symptom in the prevalent population $(24 \%)$ as compared with the incident population (12\%) might support this interpretation. Other possible explanations are that modern paraclinical tools have facilitated multiple sclerosis diagnostics in elderly people, or that a change in multiple sclerosis phenotype towards a later onset may have occurred.

There was no significant difference between the age at onset of PPMS and the age of conversion from RRMS to SPMS for prevalent cases. Interestingly, the median time to SPMS conversion added to the median age at onset for all RR/ SPMS cases gives an age at onset of secondary progression identical to that for PPMS in prevalent cases. This is in agreement with recent studies which show similar progression rates as well as other epidemiological similarities between these two clinical subtypes. ${ }^{78}$ These data support the view that RR/SPMS and PPMS are not different entities, but only variants of the same disease.

\section{Anatomical correlation between first and second attack}

An anatomical correlation between the first and second attacks has been described before. ${ }^{9}$ One possible explanation for this finding may be an increased recognition of previously experienced symptoms in the individual. However, as the second attack had to show dissemination in space-this being especially difficult for spinal attacks-we believe that the present finding of a $40 \%$ increased risk of having the first and second attack in the same anatomical region is rather an underestimation.

\section{Multiple sclerosis natural history data}

Several studies have evaluated the natural history of multiple sclerosis. A recent study from Iceland provides the hitherto most favourable description of the disease course. For example, $71 \%$ of incident Icelandic cases with a disease duration of 10 to 14 years had an EDSS of $<4 .{ }^{10}$ The corresponding figure in the present prevalence population was $61 \%$.

The most elaborate and cited study of the natural history of multiple sclerosis is a follow up of cases from 1972 to 1984 at the multiple sclerosis clinic at the University Hospital in London, Middlesex County, Ontario, Canada. One geographically based subgroup, the Middlesex County subgroup, was known to represent $90 \%$ of cases identified in a population based survey of this region and was regarded as a cross sectional subset. ${ }^{6} 11$

Figure 3 shows the distribution of disability status scale (DSS) for the London (Ontario) total population $(\mathrm{n}=1098)$, the Middlesex County subgroup $(n=196)$, and the EDSS distribution for the Västerbotten prevalence population $(\mathrm{n}=322)$. Only cases examined in 1997 to 1998 in the Västerbotten prevalence population are used for this comparison, thereby compensating for methodological differences such as onset adjusted prevalence. Median EDSS was 4.0 in this population, compared with 3.0 for the remainder, and consequently EDSS was not underestimated. The interpretation of DSS and EDSS differs for some scores but is similar for scores of 3 and 6 . Thus disability, as measured by DSS and EDSS, was aggregated into three groups: less than 3, 3 to 5, and 6 and above. The proportion of cases with EDSS/DSS less than 6 was 53\% in the Middlesex County subgroup, compared with $61 \%$ in the Västerbotten population. This difference, indicating a lesser degree of disability in the present prevalence population, was found despite the fact that data were lacking for the most disabled $10 \%$ of cases

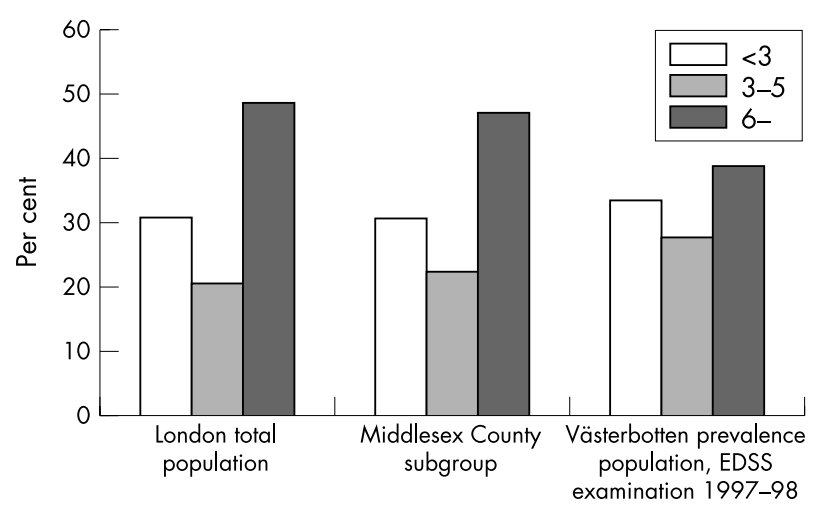

Figure 3 Disability distribution for the London (Ontario) total population (median disease duration 10 years), the Middlesex County (Ontario) subgroup (12 years), and the Västerbotten prevalence population examined in 1997 to 1998 (16 years). Disability was measured using the disability status scale (DSS) in the London, Ontario populations, and the extended disability status scale (EDSS) in the Västerbotten population.

belonging to the Middlesex County subgroup. ${ }^{6}$ More importantly, the Middlesex County subgroup had a median disease duration of merely 12 years ( 10 years in the London total population) compared with 16 years in the Västerbotten prevalence population. Figure 4 shows the difference in disease duration between the London total population, the Middlesex County subgroup at final review, and the Västerbotten prevalence population (data for all three available only for RR/SPMS).

Three variables were possibly related to the higher disability despite the shorter disease duration in London, Ontario, compared with Västerbotten. These were as follows.

The proportion of progressive multiple sclerosis from onset

As progressive multiple sclerosis generates most of the accumulated disability in a given multiple sclerosis population, the proportion of cases with this form from the onset will influence disability in that population. In the initial clinical course classification, $33 \%$ in the London total population and $30 \%$ in the Middlesex County subgroup had
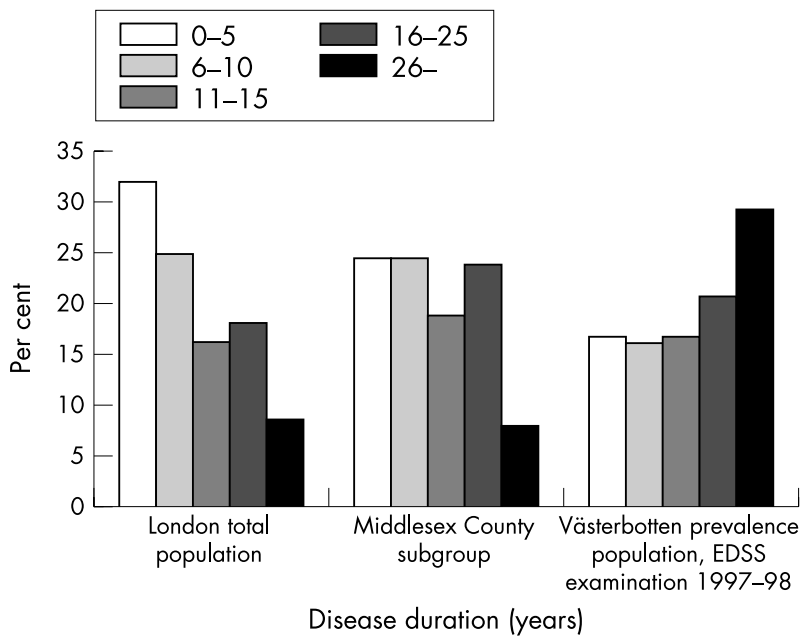

Figure 4 Comparison of disease duration for relapsing-remitting and secondary progressive multiple sclerosis cases in the London (Ontario) total population ( $n=722)$, Middlesex County (Ontario) subgroup $(n=138)$, and the Västerbotten prevalence population (only cases examined in 1997 to $1998 ; n=263$ ). 


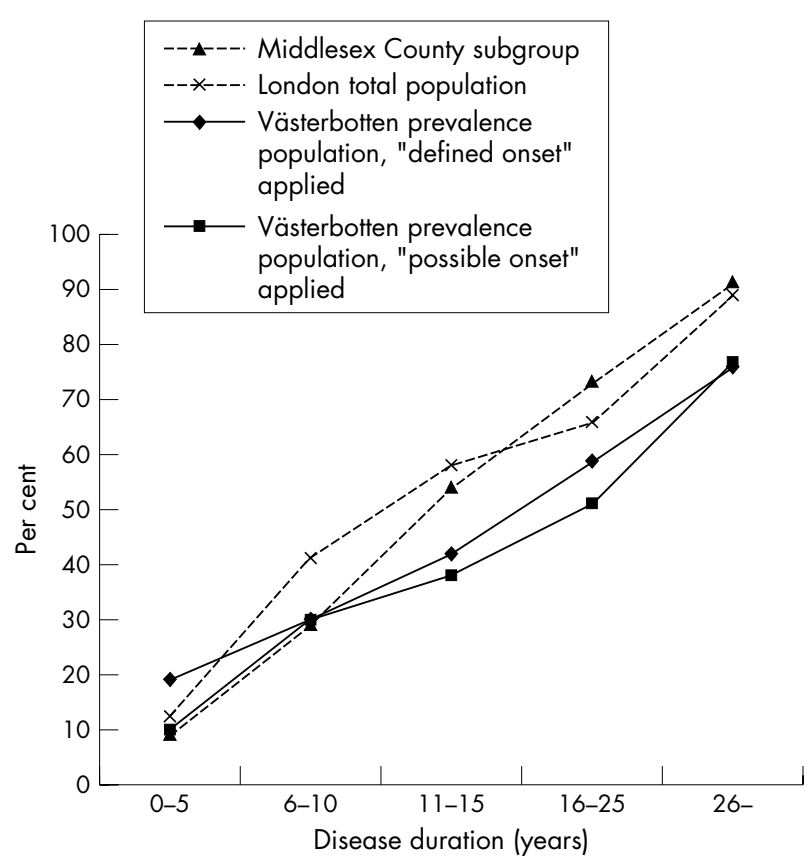

Figure 5 Percentage of relapsing-remitting cases of multiple sclerosis converting to secondary progressive multiple sclerosis, by disease duration. Data for the Middlesex County (Ontario) subgroup, the London (Ontario) total population, and Västerbotten prevalence population with "defined" and "possible onset" applied.

progressive multiple sclerosis from onset. ${ }^{6}$ The clinical course distribution in the London population has been re-evaluated using the same criteria as in the present study, but still $27 \%$ (author's estimate) are classified as PPMS or PRMS. ${ }^{12}$ The corresponding figure in the present study is 16\% (PPMS and PRMS), which is significantly lower than for the Middlesex County subgroup $(\mathrm{p}<0.001)$. The present proportion of cases with progression from onset is in accordance with findings in other elaborate epidemiological studies of multiple sclerosis. ${ }^{713}$

\section{Study population}

There is a risk that some selection bias may have occurred in the London multiple sclerosis population as it consists of cases referred to a tertiary referral centre, the London multiple sclerosis clinic. Furthermore, the crude prevalence in the Middlesex County study area $\left(95 / 10^{5}\right)$ was significantly lower than in Västerbotten $\left(154 / 10^{5}, \mathrm{p}<0.001\right) .{ }^{16}$ It is likely that the methodology used in the present population will be more likely to identify relatively benign cases.

\section{Time to SPMS conversion}

Most people with multiple sclerosis initially have attacks. However, most of the disability, if not all, is generated by disease progression. ${ }^{7}$ The proportion of cases converting from RRMS to SPMS by disease duration in the Västerbotten prevalence population is compared with the London total population and Middlesex County subgroup in fig 5. ${ }^{6}$ The proportion of RRMS cases free from progression after a long duration of disease appears to be greater in the present sample (only significant for the London total population with a disease duration of $\geqslant 26$ years when compared with the Västerbotten population with "defined onset" applied; $\mathrm{p}<0.05$ ). The relatively high proportion of progression-free RRMS cases is also in agreement with the findings in the Göteborg incidence cohort, which was evaluated after 25 years of follow up. ${ }^{13}$

Thus despite a longer disease duration the present prevalence population has less accumulated disability compared with the cross sectional Middlesex County subgroup in the London material. Whether this represents true differences of the multiple sclerosis populations or only reflects different methodology cannot be determined for certain. However, the results from studies of the natural history of multiple sclerosis are strongly dependent on the case retrieval procedure.

\section{Conclusions}

We believe that the use of multiple sources for case identification combined with personal follow up is essential to obtain reliable data on the full spectrum of the disease. Using this methodology, one important difference from previous cross sectional epidemiological studies of multiple sclerosis appears to be the better recognition of cases of comparably mild disease. We therefore suggest that multiple sclerosis may be a rather more benign disease than previously recognised.

\section{ACKNOWLEDGEMENTS}

This study was supported by grants from the Swedish Association of Neurologically Disabled and the K-O Hansson Memorial Fund.

\section{Authors' affiliations}

P Sundström, A Svenningsson, L Forsgren, Department of

Pharmacology and Clinical Neuroscience, Umeå University Hospital, Umeå, Sweden

L Nyström, Department of Public Health and Clinical Medicine, Umeå University Hospital

Competing interests: none declared

\section{REFERENCES}

1 Sundström P, Nyström L, Forsgren L. Incidence (1988-97) and prevalence (1997) of multiple sclerosis in Västerbotten County in northern Sweden. J Neurol Neurosurg Psychiatry 2003;74:29-32.

2 Poser CM, Paty DW, Scheinberg L, et al. New diagnostic criteria for multiple sclerosis: guidelines for research protocols. Ann Neurol 1983;13:227-31.

3 Kurtzk JF. Rating neurologic impairment in multiple sclerosis: an expanded disability status scale (EDSS). Neurology 1983;33:1444-52.

4 Poser CM. Onset symptoms of multiple sclerosis. J Neurol Neurosurg Psychiatry 1995; 58:253-4.

5 Lublin FD, Reingold SC. Defining the clinical course of multiple sclerosis: results of an international survey. National Multiple Sclerosis Society (USA) advisory committee on clinical trials of new agents in multiple sclerosis. Neurology 1996;46:907-11.

6 Weinshenker BG, Bass B, Rice GP, et al. The natural history of multiple sclerosis: a geographically based study. I. Clinical course and disability. Brain 1989;112:133-46.

7 Confavreux C, Vukusic S, Moreau T, et al. Relapses and progression of disability in multiple sclerosis. N Engl I Med 2000;343:1430-8.

8 Trojano $M$, Liguori $M$, Bosco Zimatore $G$, et al. Age-related disability in multiple sclerosis. Ann Neurol 2002;51:475-80.

9 Andersen O. Restricted dissemination of clinically defined attacks in an MS incidence material. Acta Neurol Scand Suppl 1980;77:1-70.

10 Benedikz J, Stefansson M, Guomundsson J, et al. The natural history of untreated multiple sclerosis in Iceland. A total population-based 50 year prospective study. Clin Neurol Neurosurg 2002; 104:208-10.

11 Hader WJ, Elliot M, Ebers GC. Epidemiology of multiple sclerosis in London and Middlesex County, Ontario, Canada. Neurology 1988;38:617-21.

12 Kremenchutzky M, Cottrell D, Rice G, et al. The natural history of multiple sclerosis: a geographically based study. 7. Progressive-relapsing and relapsing-progressive multiple sclerosis: a re-evaluation, Brain 1999;122:1941-50.

13 Runmarker B, Andersen O. Prognostic factors in a multiple sclerosis incidence cohort with twenty-five years of follow-up. Brain 1993;116:117-34. 